www.jmscr.igmpublication.org Impact Factor 5.84

Index Copernicus Value: 71.58

ISSN (e)-2347-176x ISSN (p) 2455-0450

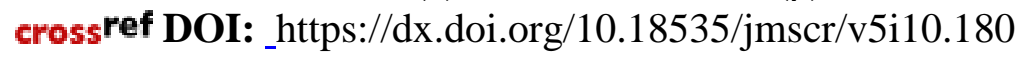

Journal Of Medical Science And Clinical Research

\title{
A Study on Clinical Determinants and one year Follow-Up of Dementia and Mild Cognitive Impairment Following Ischemic Stroke
}

\author{
Authors \\ Murugapandian Nagarajan ${ }^{1}$, Arunan Subbiah², Palani Kannan ${ }^{3}$ \\ ${ }^{1}$ Senior Assistant Professor of Neurology, Govt. Kilpauk Medical College, Chennai, India \\ ${ }^{2}$ Associate Professor of Neurology, Govt. Kilpauk Medical College, Chennai, India \\ ${ }^{3}$ Resident, Govt. Kilpauk Medical College, Chennai, India \\ Corresponding Author \\ Murugapandian Nagarajan
}

Senior Assistant Professor of Neurology, Govt. Kilpauk Medical College, Chennai-10, India

Email: neuropandian@gmail.com, Tel:+91-9443921569

\begin{abstract}
Background: Vascular cognitive impairment (VCI) is poised to become the silent epidemic of the 21st century and contribute significantly to mortality, disability, and decreased quality of life.

Aim: To investigate the frequency and clinical determinants of vascular dementia (VD) and Vascular mild cognitive impairment (VMCI) following ischemic stroke. The one year follow-up was done to assess the prognosis.

Materials and Methods: A prospective study was conducted among 100 consecutive patients aged $\geq 40$ years with the diagnosis of ischemic stroke within previous 2 - 3 months. The subjects were be subjected to structured medical and neurological history based on review of all available hospital charts, interview of the subject and a knowledgeable informant, and a structured clinical, neurological, neuropsychological, and neuroradiological evaluation. Patients will be retested at 3, 6, and 12 months after stroke.

Results: $60 \%$ of stroke patients were diagnosed as VCI. 23 subjects were diagnosed as VD. The prevalence of VMCI was $37 \%$ and forty percent of the patients had no cognitive impairment. An annual conversion rate from 'VMCI' to 'VD was $8 \%$. The annual rate of deterioration from 'No Cognitive Impairment' to 'VMCI' was 10\%. Conclusion: This study supports the high prevalence of dementia and mild cognitive impairment following stroke, especially in older individuals, and highlights the importance of cognitive reserve.

Keywords: Ischemic stroke, vascular dementia, Vascular cognitive impairment, Vascular mild cognitive impairment.
\end{abstract}

\section{Introduction}

Cognitive impairment due to cerebrovascular disease is termed "Vascular Cognitive Impairment" (VCI) and forms a spectrum that includes vascular dementia (VD) and milder forms of cognitive impairment referred to as vascular mild cognitive impairment (VMCI). ${ }^{[1]}$ While VD is the second most common cause of dementia, the milder form VMCI is much more common. Nearly half of individuals with VMCI convert to dementia after five years. Vascular cognitive disorders are poised to become the silent epidemic of the 21 st century and contribute significantly to mortality, disability, and decreased quality of life. 
It is now clear that VCI is not a single entity, but represents a complex neurological disorder that occurs as a result of interaction between vascular risk factors and brain parenchymal changes such as macro and micro infarcts, haemorrhages, white matter changes, and brain atrophy occurring in an ageing brain.

Factors that determine progression of milder form VMCI to dementia are not well understood. Since VCI is amenable to prevention and treatment, there is a pressing need to identify factors that protect or predispose to it.

Dementia is common sequelae of stroke with a frequency ranging from $16 \%$ to $32 \% .{ }^{[2]}$ In a more recent epidemiological study, the 10-year risk of dementia after stroke was estimated at 19.3\%, compared to $11.0 \%$ in non-stroke controls. ${ }^{[3]}$ This twofold increase in dementia following stroke is in contrast with a nine fold increase reported in some previous studies. The large variation in rates is accounted for by variations in the populations studied as well as the criteria used for the diagnosis of dementia. As the definition of VD is currently being refined, it is timely that these studies be revisited. One aspect of this reassessment is the consideration of VMCI in stroke patients, which has thus far received little attention.

Most studies on post stroke cognitive impairment have focused on dementia. Bowler and Hachinski a decade ago made warnings about the limitations of the diagnostic category of VD and coined the term VCI to refer to any cognitive impairment related to cerebrovascular disease. Detection of cognitive impairment has two difficulties: a neuropsychologic battery fitted to the VCI profile is yet to be established and limits from normal cognition are still undefined. Also, the evidence of the vascular origin of cognitive impairment is difficult to ascertain.

\section{Aim}

- To investigate the frequency and clinical determinants of dementia and mild cognitive impairment following ischemic stroke. The one year follow-up was done to assess the prognosis.

\section{Materials and Methods}

A prospective study is conducted to evaluate the frequency and clinical determinants of dementia and mild cognitive impairment among 100 ischemic stroke patients.

Inclusion criteria: 100 consecutive patients aged $\geq 40$ years with the diagnosis of ischemic stroke within previous 2 - 3 months.

Exclusion criteria: 1.Prestroke dementia 2.No baseline CT3.Neurological disorder other than the qualifying event (e.g.- Parkinson's disease) 4.Major psychiatric disorder which could lead to cognitive deficits 5.Severe aphasia6.Severe hearing impairment7.Those not speaking Tamil or English Those not speaking Tamil or English

An ischemic stroke is defined as 'rapidly developing clinical signs of focal (or global) disturbance of cerebral function, with symptoms lasting 24 hours or longer, with no apparent cause other than of vascular origin' in which a brain CT or MRI scan does not show intracranial haemorrhage.

An interval of 2-3 months following stroke onset for neuropsychological Testing is chosen to allow sufficient time for the acute stroke effects to subside.

The subjects were be subjected to structured medical and neurological history based on review of all available hospital charts, interview of the subject and a knowledgeable informant, and a structured clinical, neurological, neuropsychological, and neuroradiological evaluation. Patients will be retested at 3, 6, and 12 months after stroke. At the time of entry, the following data were collected : (1) sex; (2) age (categorized as between 40 and 59, between 60 and 69 , and $\geq$ 70); (3)education; (4) cerebrovascular risk factors, including cigarette smoking, hypertension (treatment or two or more measurements before stroke of systolic pressure 140 or diastolic pressure $90 \mathrm{~mm} \mathrm{Hg}$ ), diabetes (treatment or fasting blood glucose $>7.8 \mathrm{mmol} / \mathrm{L}$ before stroke), 
hypercholesterolemia (total cholesterol >6.2 $\mathrm{mmol} / \mathrm{L}$ ), ischemic heart disease (previous myocardial infarction or angina), previous history of transient ischemic attack or stroke, and atrial fibrillation; and (5) presence of aphasia in the acute phase of stroke.

In depth probes in the history for risk factors and features suggesting of pre-morbid cognitive impairment were attempted. Significant past medical history if any were noted. A thorough clinical examination was performed at the time of first visit. A routine screening, which included blood sugar, urea, serum creatinine, lipid profile, four-vessel doppler study, electrocardiogram, and echocardiogram were done at the time of admission.

Neuropsychological Assessment was done with VD Battery. The VD Battery assesses six cognitive domains: attention, language, verbal memory, visual memory, visuo construction, visuomotor speed and executive functions. The Mini-Mental State Examination (MMSE) was also administered together with the VD Battery.

\begin{tabular}{|l|c|}
\hline Domains & Neuropsychological tests \\
\hline Attention & Digit Span Forward \\
\hline Language & $\begin{array}{c}\text { Modified Boston Naming Test } \\
\text { Verbal Fluency Test ( Category } \\
\text { naming of animals in 1 minute ) }\end{array}$ \\
\hline Memory & RAVLT \\
\hline Visuoconstruction & $\begin{array}{c}\text { Clock Drawing Test } \\
\text { Crossing-pentagons item from MMSE }\end{array}$ \\
\hline Visuomotor Speed & Digit Cancellation Task \\
\hline $\begin{array}{l}\text { Executive } \\
\text { function }\end{array}$ & Trail-making test B \\
\hline
\end{tabular}

The patients' performance was compared with that of a group of normal control subjects from previous studies.

Neuroradiological assessment was done with cranial imaging, either computed tomography (CT) scan or magnetic resonance imaging (MRI). MRI was done in 61 of 100 patients, and all 100 patients underwent CT scan. Infarcts were identified on neuroimaging and classified as cortical, subcortical, or borderzone. Subcortical infarcts were further classified as lacunar and nonlacunar infarcts. Stroke volume was calculated by using the formulae $0.5 \times \mathrm{A} \times \mathrm{B} \times \mathrm{C}$.
For dementia (VD) diagnosis, a subject must have fulfilled NINDS-AIREN criteria (the National Institute for Neurological Disorders and StrokeAssociation Internationale pour la Recherche et a'Enseignementen Neurosciences). For a diagnosis of VMCI, the subject must have definite impairment in one domain and the functional decline criterion for VD was not met. Impairment in a cognitive domain was defined as definite impairment ( $>5$ th percentile) on at least one test. VD was classified based on brain imaging as cortical VD in patients with multiple cortical infarcts, as subcortical VD when VD was associated with subcortical infarcts and/or white matter hyperintensities, or as cortical-subcortical VD in those with a combination of cortical infarcts and discrete subcortical infarcts/ white matter hyperintensities.Strategic infarct dementia was diagnosed when dementia could be explained by a single, strategically placed infarct. Subcortical VD was further classified depending on the predominant lesion type as subcortical VD due to subcortical infarcts, white matter hyperintensities, or a combination of the two.

After informed consent, patients were reexamined 3, 12, and 24 months after stroke. In these visits, physical and neurological examinations, functional assessment, neuropsychologic battery, and diagnosis of Dementia and VMCI were performed.

\section{Results}

$60 \%$ of stroke patients were diagnosed as VCI. 23 subjects were diagnosed as VD. Two subjects in the age range 40-59 years were diagnosed with VD, whereas 9 in the 60-69 years, and 12 in the $\geq 70$ years group had this diagnosis. The prevalence of VMCI was $37 \%$ and forty percent of the patients had no cognitive impairment. (Table.1 and 2)

Of the 176 patients with ischemic stroke screened for study suitability, 119 met inclusion and exclusion criteria and were entered into the study. The major reasons for exclusion, in order of frequency, were presented in Figure.1. 
By the time of detailed assessment at 3 months following the ischemic stroke, 8 were lost to follow-up (4 relocated outside Chennai and was not contactable, 3 died, 1 was refused for further follow-up). Of the 111 patients 100 completed follow-up at 1 year and 11 were lost to follow-up (2 died, 1 was reclassified as a patient with possible Alzheimer's disease, and 8 were not contactable).

The demographic and functional characteristics of the study sample were presented in Table.3. Mean age was 66.3 years (range 48 to 85 ). $29 \%$ of female patients were included in this study. Among the females fifty five percent had cognitive impairment whereas sixty one percent of male patients had the diagnosis of cognitive impairment. Stroke subjects had a mean age of 66.3 years and $71 \%$ were men. The numbers in the age bands $40-59,60-69$, and $\geq 70$ years were 36 , 43 , and 21, respectively. The putative cerebrovascular risk factors were presented in table 4. When the CT scans were carefully analysed, 68 of patients had brain infarcts, which were large infarcts in 24 patients. (Table.5 and Figure.2)

Impaired subjects, those with VD or VMCI, were older but there were no sex differences in the two groups. They had 1.5 years less education than the non-impaired subjects. The two groups did not differ significantly on any particular cerebrovascular risk factor except that impaired patients had a higher overall number of risk factors in comparison to non-impaired patients. The two groups did not differ significantly on the side of stroke, whether the strokes involved the cerebral hemisphere or brain stem/cerebellum, and large or lacunar strokes. VD subjects had a slightly but significantly lower score on Barthel Index, indicating greater disability.

The VMCI group did not differ significantly from VD on age, sex, and education but, as expected, the VMCI had higher scores on the Mini Mental Status Examination, Barthel Index, and Blessed functional activity scale. Cerebrovascular risk factors and their numbers were similar in the two groups.

Twenty three subjects were diagnosed to have VD according to NINDS-AIREN criteria. Among these $43 \%$ had subcortical dementia, the commonest type of VD found in this study. Another 26\% percent had cortical-subcortical type of dementia the second commonest type. Two patients had VD due to strategic infarct one due to bilateral thalamic infarct and another due to anterior opercular syndrome. (Figure.3) When cognitive impairment was examined by specific vascular territory and laterality (table 6), differences were less obvious, in part because of small samples in some of the subgroups. Overall, cognitive impairment appeared most frequently with infarcts in the left anterior and posterior cerebral artery territories and least frequently with infarcts in the vertebrobasilar artery territory. Examining vascular distribution independently of hemispheric side, the proportion of infarcts in the anterior cerebral artery territory was higher among those with cognitive impairment (7.6\%) compared with those without (3.4\%). Comparing left-sided, right-sided and vertebrobasilar strokes, left-sided damage was more common among those with cognitive impairment $(n=28)$ compared with those without $(\mathrm{n}=17)$.

Baseline and 1-year follow-up classification of the 100 patients:The baseline (3 months) and 12months results of various clinical parameters are given in Table.7.

Of the 100 stroke patients who were reassessed, the baseline and 1-year follow-up classification are tabulated in Table. Eighty patients had the same classification as at baseline. Of the 20 patients who had a changed classification, the majority were 'VMCI' at baseline with 10 improving to 'No Cognitive Impairment' and three deteriorating to 'VD'-an annual conversion rate (from 'VMCI' to 'VD') of $8 \%$. The annual rate of deterioration from 'No Cognitive Impairment' to 'VMCI' was $10 \%$ but none of this group of patients became 'demented'. (Table .8) 
Table. 1. Prevalence of Vascular Cognitive Impairement

\begin{tabular}{|l|l|}
\hline No cognitive Impairment & 40 \\
\hline VMCI & 37 \\
\hline VD & 23 \\
\hline
\end{tabular}

Table .2. Prevalence of Impairment in various age groups

\begin{tabular}{|l|c|c|c|c|}
\hline & $40-59$ & $60-69$ & $\geq 70$ & Total \\
\hline VMCI & 6 & 26 & 5 & 37 \\
\hline VD & 2 & 9 & 12 & 23 \\
\hline NCI & 28 & 8 & 4 & 40 \\
\hline
\end{tabular}

Table .3. The demographic and functional characteristics

\begin{tabular}{|l|c|c|c|c|}
\hline & $\begin{array}{c}\text { All patients } \\
(\mathrm{n}=100)\end{array}$ & $\begin{array}{c}\text { NCI } \\
(\mathrm{n}=40)\end{array}$ & $\begin{array}{c}\text { VMCI } \\
(\mathrm{n}=37)\end{array}$ & $\begin{array}{c}\text { VD } \\
(\mathrm{n}=23)\end{array}$ \\
\hline Age, years & 66.3 & 61.5 & 67.7 & 72.3 \\
\hline Female/Male & $29 / 71$ & $13 / 27$ & $8 / 29$ & $8 / 15$ \\
\hline Education, years & 8.6 & 9.9 & 8.2 & 6.9 \\
\hline MMSE & 27.9 & 29.1 & 28.0 & 25.8 \\
\hline Barthel Index & 85 & 90 & 85 & 65 \\
\hline
\end{tabular}

Table .4.Risk Factors.

\begin{tabular}{|l|c|c|c|c|}
\hline Risk factors & Stroke patients & NCI & VMCI & VD \\
\hline Hypertension & 56 & 23 & 19 & 14 \\
\hline Hyper Cholesterolemia & 34 & 13 & 15 & 6 \\
\hline Smoker & 51 & 25 & 18 & 8 \\
\hline Diabetes mellitus & 14 & 5 & 5 & 4 \\
\hline Previous TIA & 18 & 5 & 8 & 5 \\
\hline Previous stroke & 12 & 4 & 5 & 3 \\
\hline Previous AMI & 17 & 6 & 7 & 4 \\
\hline Previous angina & 19 & 6 & 6 & 7 \\
\hline Atrial fibrillation & 11 & 4 & 3 & 4 \\
\hline Mean CVRF score & 2.5 & 2.2 & 2.6 & 2.8 \\
\hline
\end{tabular}

Table .5. Clinical and CT brain characteristics of the sample

\begin{tabular}{|l|c|c|c|}
\hline & NCI $(\mathrm{n}=40)$ & VMCI $(\mathrm{n}=37)$ & VD $(\mathrm{n}=23)$ \\
\hline Clinical stroke & & & \\
Laterality (right sided) & 18 & 19 & 11 \\
Lacunar infarct & 13 & 9 & 5 \\
Prior CVA & 4 & 5 & 3 \\
\hline CT Brain Infarct Volume, $\mathrm{mm}^{3}$ & 1430 & 860 & 4300 \\
\hline
\end{tabular}

Table.6. Cognitive impairment and Stroke territory

\begin{tabular}{|l|c|c|}
\hline \multirow{2}{*}{ Stroke territory } & \multicolumn{2}{|c|}{ Cognitive impairment } \\
\cline { 2 - 3 } & Present $(\mathrm{n}=60)$ & Absent $(\mathrm{n}=40)$ \\
\hline Left ICA & 1 & 0 \\
\hline Right ICA & 0 & 1 \\
\hline Left ACA & 3 & 2 \\
\hline Right ACA & 2 & 2 \\
\hline Left MCA & 19 & 13 \\
\hline Right MCA & 18 & 10 \\
\hline Left PCA & 6 & 4 \\
\hline Right PCA & 5 & 2 \\
\hline Vertebrobasilar & 6 & 6 \\
\hline
\end{tabular}


Table .7. Baseline and Follow-up results of clinical parameters

\begin{tabular}{|l|c|c|c|c|c|c|}
\hline Tests & \multicolumn{2}{|c|}{ NCI } & \multicolumn{2}{c|}{ VMCI } & \multicolumn{2}{c|}{ VD } \\
\hline & $\begin{array}{c}\text { At 3 } \\
\text { months }\end{array}$ & $\begin{array}{c}\text { At 12 } \\
\text { months }\end{array}$ & $\begin{array}{c}\text { At 3 } \\
\text { months }\end{array}$ & $\begin{array}{c}\text { At 12 } \\
\text { months }\end{array}$ & At 3 months & $\begin{array}{c}\text { At 12 } \\
\text { months }\end{array}$ \\
\hline MMSE & 29.1 & 29 & 28.0 & 28.6 & 25.8 & 22.2 \\
\hline Barthel Index & 89.9 & 92.4 & 79.1 & 79.9 & 68.7 & 71.5 \\
\hline BDI & 9.7 & 9.6 & 13.6 & 13.8 & 15.9 & 15.8 \\
\hline Digit span forward & 6.1 & 6.6 & 5.0 & 6.1 & 4.2 & 4.9 \\
\hline $\begin{array}{l}\text { Trail-making } \\
\text { Test B }\end{array}$ & 123 & 98 & 191 & 170 & 262 & 201 \\
\hline Modified Boston Naming Test & 13.8 & 14.1 & 10.2 & 10.7 & 7.1 & 9.1 \\
\hline
\end{tabular}

Table .8. Baseline and Follow-up classification of Cognitive impairment.

\begin{tabular}{|l|l|c|c|c|c|}
\hline \multicolumn{2}{|c|}{} & \multicolumn{4}{|c|}{ Classification at 1-year follow-up } \\
\cline { 3 - 6 } \multicolumn{2}{|c|}{} & NCI & VMCI & VD & Total \\
\hline Classification at baseline & NCI & 36 & 4 & 0 & 40 \\
\cline { 2 - 6 } & VMCI & 10 & 24 & 3 & 37 \\
\cline { 2 - 6 } & VD & 0 & 3 & 20 & 23 \\
\hline
\end{tabular}

Figure .1. Reasons for exclusion from study

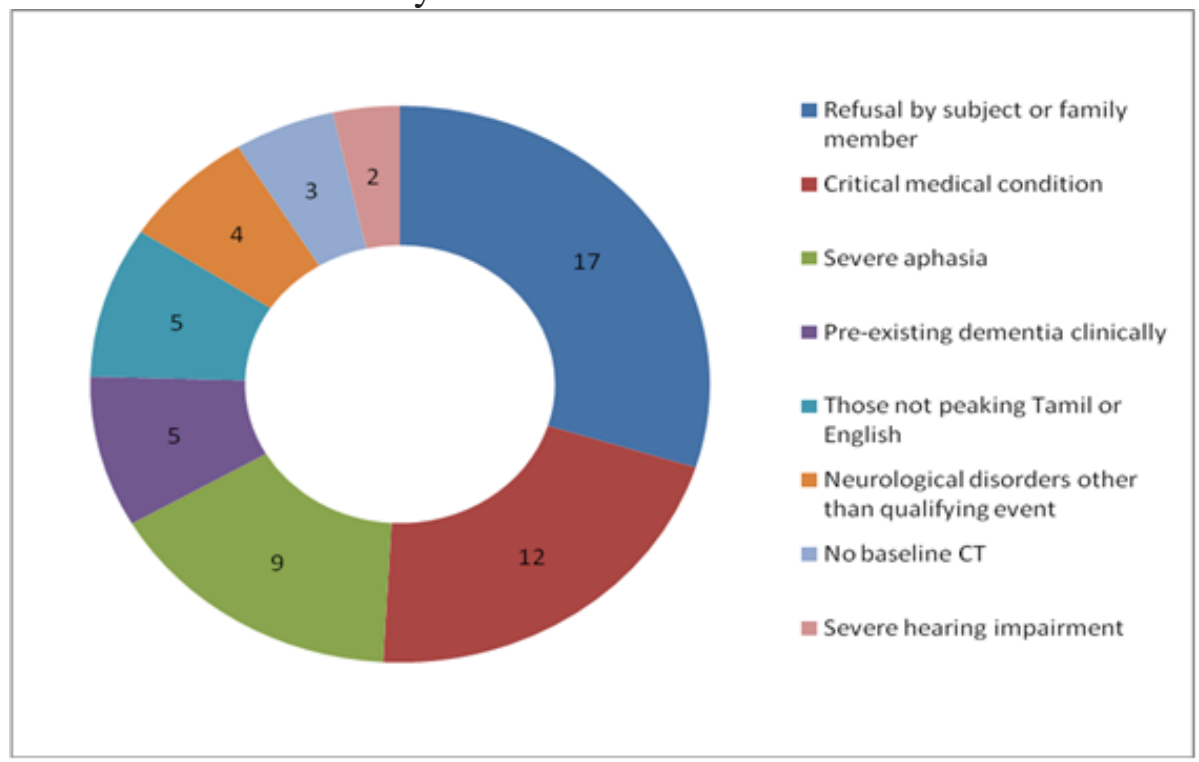

Figure.2. Stroke Volume

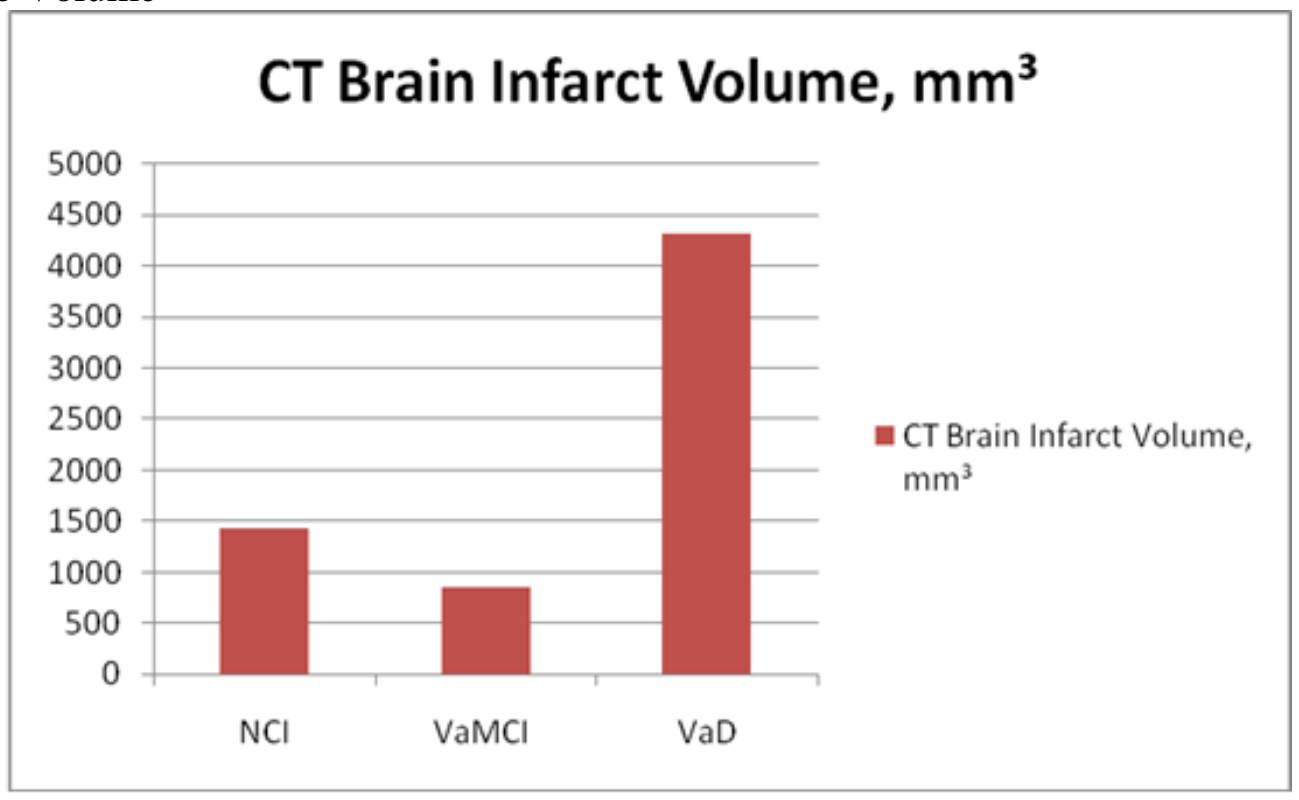


Figure.3. Subtypes of Vascular Dementia

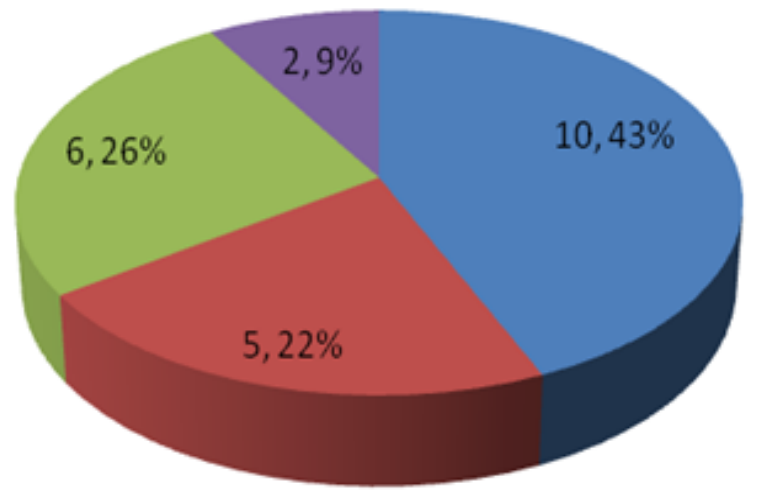

- Subcortical

- Cortical

- Cortical-Subcortical

- Strategic infarct

\section{Discussion}

The prevalence rate of VD is $23 \%$ in a well characterised cohort of stroke subjects examined 3 months after the index event. All cases of VD, bar two, were diagnosed in those over 60 years. The figures are consistent with previous reports, with one previous study ${ }^{[4,5]}$ reporting a rate of $26.4 \%$ in individuals aged 60-90 years, and another study [6] reporting a rate of $25 \%$ in individuals 55-85 years old.

Epidemiologic studies from India have shown that VD is a common form of dementia, in contrast to the West. This finding may be related to the overall reduced life span in Indians and the increasing burden of cardiovascular disease, including stroke. Recent observations of the decreased prevalence of VD in Japan, attributed to decreased incidence of stroke and westernization, support this hypothesis.

Age was a significant determinant of prevalence in this study only when more than 60 years was used as the cut-off, i.e. the prevalence rate did not increase after the age of 60 years. In the New York study, subjects in their 60s had a lower rate than those 70 years and older. When age was entered into logistic regression along with other possible determinants of dementia, it was no longer significant. This is possibly because age is correlated with cerebrovascular risk factors as well as brain imaging parameters such as atrophy and White mater hyperintensities. It can be concluded that a stroke in an older person is more likely to produce dementia than in someone less than 60 years. This is considered to be due to the additional cerebrovascular pathology in older brains, which may be due to previous infarctions and non-infarct ischemic changes. The role of Alzheimer-type pathology in older brains is another important factor, with post mortem examination necessary to determine its relative contribution.

The prevalence rate of VD in this study compares well with other hospital-based populations ${ }^{[4,5,6]}$. The rate of dementia was $19.3 \%$ in stroke patients compared to $11.0 \%$ in controls, suggesting a twofold increase in risk of stroke over a 10- year period in the community-based sample $\mathrm{e}^{[7]}$. The NINDS-AIREN ${ }^{[8]}$ criteria used in this study may also have played a role as they are insensitive to subcortical dementia. The reason that I chose these criteria was to minimize the inclusion of patients with Alzheimer's disease or mixed dementia in this VD group. 
In this study, subcortical dementia due to multiple lacunar infarcts and/or WMHs was the most common subtype of VD, whereas dementia due to multiple cortical infarcts and single strategic infarcts was less common. Similar patterns of VD have been reported in Japanese and Western populations.

The definition of VD used is an important consideration in the interpretation of prevalence rates. The construct of VD is still evolving and the currently available criteria sets have low correspondence with each other. The criteria used in this study differed from those used in other studies in that memory impairment was necessary for the diagnosis of VD. This is similar to the DSM-IV criteria ${ }^{[9]}$ but different from the ADDTC ${ }^{[8]}$. Unlike the ADDTC criteria, however, the presence of two or more ischemic strokes or one or infarcts on CT or T 1 -weighted MRI was not necessary, and extensive white matter disease was sufficient for the diagnosis. Another feature of my diagnoses was that the neuropsychological and functional criteria were operationalised, and the significance of the vascular pathology was based on CT or MRI scans. CT scans were available for all patients. However, the decision whether the vascular pathology on brain scans was sufficient to account for the cognitive impairment was based on 'clinical judgement'. The presence of an infarct was not considered necessary if the subject had extensive white matter pathology, although most subjects had a combination of the two kinds of lesions. Furthermore, memory impairment was not an essential criterion for the diagnosis of impairment or dementia. Applying the nonmemory impairment criterion to our definition of dementia would have changed the prevalence considerably. Six of the VMCI subjects had marginal or definite memory impairment in either verbal or non-verbal domain, and would have met the NINDSAIREN criteria.

This study also reports the prevalence of MCI in this sample, presumably again of vascular origin. More than one third of stroke subjects met the criteria for MCI, and the combined VD and VMCI groups accounted for nearly $60 \%$ of the sample. This suggests that the majority of stroke subjects have cognitive impairment, with about a quarter reaching the threshold for dementia diagnosis. I was interested in knowing whether the determinants for dementia were qualitatively or quantitatively different from those for VMCI.

Previous studies have identified some strokerelated factors as determinants of dementia, in particular lacunar infarcts, left-sided lesions and hemispheric infarcts ${ }^{[4,10]}$. A major dominant stroke syndrome and dysphasia have also been related to dementia ${ }^{[10,11]}$. These findings have, however, not been consistent, with other studies failing to support these relationships ${ }^{[12]}$, including this study. Since this study necessitated a demanding assessment schedule, it is likely that many severely ill subjects were excluded. I also excluded subjects with severe aphasia or nonfluency in Tamil or English because of the difficulty in obtaining informed consent and assessing neuropsychological function. This may have contributed to the lack of a left-hemispheric bias in this dementia subjects.

Hypertension, diabetes, atrial fibrillation and other recognised cerebrovascular risk factors have emerged as independent risk factors for dementia in some studies but not others. In the Framingham Study, after stroke had been accounted for, exposure to individual risk factors did not alter the hazard ratio. The analysis in this study did suggest that overall cerebrovascular risk factor exposure was associated with cognitive impairment, but it was not significant in the combined regression analysis.

In the brain imaging parameters, this study assessed only total stoke volume. It was significant (OR 1.8). Brain atrophy in this study, being measured after the stroke, does not accurately reflect baseline atrophy since it is confounded by the stroke itself. I had hypothesised that stroke volume and number would be independent predictors of dementia, and this was only partially borne out. 
Follow-up: A more recent analysis of the Canadian Study of Health and Ageing cohort, showed that nearly half of those who had "VCI without dementia" developed dementia within 5 years ${ }^{[13]}$. These findings are similar to previous estimates of the likelihood to progression to dementia in Mild Cognitive Impairment (MCI) individuals $^{[14,15]}$. Moreover, those who are 'cognitively impaired but not demented' had a high risk of converting to dementia, comparable to the results from the Canadian Study of Health and Ageing cohort.

\section{Conclusion}

This study supports the high prevalence of dementia and mild cognitive impairment following stroke, especially in older individuals, and highlights the importance of cognitive reserve. The contribution of cerebrovascular risk factors is not independent of the stroke risk. Subcortical dementia was the most frequent subtype of VD in our hospital-based series. Stroke volume is a significant determinant of post-stroke dementia. This study suggests that cognitive performance in stroke patients may change over time. It also suggests that progression of cognitive impairment is common after stroke.

\section{Acknowledgement}

We the authors sincerely thank the participants, staffs, paramedics and statistician for their cooperation and their contribution towards this study.

\section{Conflict of interest}

All authors contributed equally in developing the manuscript.

\section{References}

1. Hachinski VC, Bowler JV. Vascular dementia: Diagnostic criteria for research studies. Neurology 1993;43:2159-60.

2. Tatemichi TK, Foulkes MA, Mohr JP, Hewitt JR, Hier DB, Price TR, et al. Dementia in stroke survivors in the Stroke
Data Bank cohort. Prevalence, incidence, risk factors, and computed tomographic findings. Stroke 1990;21(6):858-66.

3. Ivan CS, Seshadri S, Beiser A, Au R, Kase CS, Kelly-Hayes M, Wolf PA: Dementia after stroke: the Framingham Study: Stroke 2004; 35: 1264-1269.

4. Tatemichi TK, Desmond DW, Paik M, Gropen TI, Stern Y, Sano M, Remien R, Williams JBW, Mohr JP, Mayeux R: Clinical determinants of dementia related to stroke. Ann Neurol 1993; 33: 568-575.

5. Desmond DW, Moroney JT, Paik MC, Sano M, Mohr JP, Aboumatar S, Tseng CL, Chan S, Williams JBW, Remien RH, Hauser WA, Stern Y: Frequency and clinical determinants of dementia after ischemic stroke. Neurology 2000;54: 1124-1131

6. Pohjasvaara T, Erkinjuntti T, Ylikoski R, Hietanen M, Vataja R, Kaste M: Clinical determinants of poststroke dementia. Stroke 1998; 29: 75-81.

7. Ivan CS, Seshadri S, Beiser A, Au R, Kase CS, Kelly-Hayes M, Wolf PA: Dementia after stroke: the Framingham Study: Stroke 2004; 35: 1264-1269.

8. American Psychiatric Association: Diagnostic and statistical manual of mental disorders, $4^{\text {th }}$ ed. Washington: American Psychiatric Association, 1994.

9. Chui HC, Victoroff JI, Margolin D, Jagust W, Shankle R, Katzman R: Criteria for the diagnosis of ischemic vascular dementia proposed by the State of California Alzheimer's Disease Diagnostic and Treatment Centres. Neurology 1992; 42: 473-480.

10. Pohjasvaara T, Erkinjuntti T, Ylikoski R, Hietanen M, Vataja R, Kaste M: Clinical determinants of poststroke dementia. Stroke 1998; 29: 75-81.

11. Ladurner G, Iliff LD, Lechner H: Clinical factors associated with dementia in 
ischemic stroke. J NeurolNeurosurg Psychiatry 1982; 45.

12. Pohjasvaara T, Vataja R, Leppavuori A, Erkinjuntti T: Dementia poststroke. Psychogeriatrics 2001; 1: 88-99.

13. Wentzel C, Rockwood K, MacKnight C, Hachinski V, Hogan DB, Feldman H, et al. Progression of impairment in patients with vascular cognitive impairment. Neurology 2001:28; 57(4):714- 6.

14. Petersen RC, Smith GE, Waring SC, Ivnik RJ, Tangalos EG, Kokmen E. Mild cognitive impairment: clinical characterization and outcome.ArchNeurol 1999;56:303- 8.

15. Ritchie K, Touchon J. Mild cognitive impairment: conceptual basis and current nosological status. Lancet 2000;355:2258. 